\title{
Publisher Correction: ChromID identifies the protein interactome at chromatin marks
}

Rodrigo Villaseñor (D), Ramon Pfaendler (D), Christina Ambrosi, Stefan Butz, Sara Giuliani, Elana Bryan, Thomas W. Sheahan, Annika L. Gable, Nina Schmolka, Massimiliano Manzo, Joël Wirz, Christian Feller, Christian von Mering (D), Ruedi Aebersold (1), Philipp Voigt and Tuncay Baubec (1)

Correction to: Nature Biotechnology https://doi.org/10.1038/s41587-020-0434-2, published online 2 March 2020.

In the version of this article initially published online, an extra label, reading ARD2, was inserted on the horizontal axis of Fig. 3d between ZNF512B and JARD2. As a result, the labels were misaligned with the image grid. The error has been corrected in the print, PDF and HTML versions of the article.

Published online: 17 March 2020

https://doi.org/10.1038/s41587-020-0484-5

๑ The Author(s), under exclusive licence to Springer Nature America, Inc. 2020

\section{Publisher Correction: The Extended Polydimensional Immunome Characterization (EPIC) web-based reference and discovery tool for cytometry data}

Joo Guan Yeo (D), Martin Wasser (D), Pavanish Kumar, Lu Pan D, Su Li Poh, Fauziah Ally, Thaschawee Arkachaisri (D, Amanda Jin Mei Lim, Jing Yao Leong DD, Liyun Lai, Kee Thai Yeo (D), Elene Seck Choon Lee, Camillus Jian Hui Chua ID, Anis Larbi, Ma Shwe Zin Nyunt, Tze Pin Ng, Sabrina Chiesa, Marco Gattorno, Alberto Martini, Bhairav Suryakant Paleja, Charles-Antoine Dutertre, Jinmiao Chen (D), Katherine Nay Yaung, Swee Ping Tang, Sue Kheng Ng, Chee Fu Yung, Angela Yun June Tan, Shu Ying Lee, Florent Ginhoux (D) and Salvatore Albani (D)

Correction to: Nature Biotechnology https://doi.org/10.1038/s41587-020-0532-1, published online 21 May 2020.

In the version of this article initially published online, a portion of the image in Fig. $2 \mathrm{c}$ was shifted relative to the remainder. The error has been corrected in the print, PDF and HTML versions of the article.

Published online: 28 May 2020

https://doi.org/10.1038/s41587-020-0574-4

(c) The Author(s), under exclusive licence to Springer Nature America, Inc. 2020 